\title{
Optimal Solutions to Reduce Medical Waste
}

\author{
Chen-Lin Lee and Jin-Cherng Chen
}

\begin{abstract}
Medical waste (MW), from its creation to its final treatment, is currently a topic of great concern to society. Pollution created by MW not only has an adverse effect on human health but also harms the environment. This study developed a basic model premised on sustainable operation and environmental protection. This model provides concrete data for discussion concerning the creation and sources of MW and its harm to the environment. The government must impose fixed-rate "pollution taxes" on polluters to achieve justice. The amount of pollution emitted from various sources was calculated by using mathematical models and contingency plans were implemented for pollution control and pollution reduction to derive methods that can effectively reduce and inhibit pollution. The mathematical models can hopefully satisfy manufacturers, consumers, and the government, enabling the health care industry to achieve sustainable operation and optimized environmental protection.
\end{abstract}

Index Terms-Medical waste (MW), environment, pollution.

\section{INTRODUCTION}

Medical waste (MW), from its creation to its final treatment, is currently a topic of great concern to society. Pollution created by MW during temporary storage, transport, or treatment is harmful not only to human health but also the environment. In Taiwan, the generation of MW by hospital facilities has rapidly increased over the past decade. Data from the Environmental Protection Administration website (http://www.epa.gov.tw) show that in 2012, domestic MW totaled 90,000 tons. Reports from the World Health Organization [1] indicated that approximately $10 \%$ to $25 \%$ of the substances in MW are infectious or harmful. In other words, because MW contains not only infectious but also harmful substances, it must be effectively controlled. Failure to do so might lead to human exposure to hazardous environments. The infectious MW generated from sources including health institutes, medical centers, and healthcare facilities that produce MW includes human anatomical and surgical waste, pathological waste, sharp contaminated tools, and materials contaminated with blood and bodily fluids [2], [3]. Because MW sources vary, only medical supplies used during the medical treatment process and subsequently turned to MW were investigated.

A number of studies have already introduced methods to effectively treat MW, which have also been used in practice. Among these methods, the use of incinerators is still the most

Manuscript received May 4, 2014; revised July 7, 2014.

Chen-Lin Lee is with the Department of Dalin Tzu-Chi General Hospital, No.2, Min-Sheng Road, Dalin Town, Chia-yi 622, Taiwan (e-mail: milk8310@yahoo.com.tw).

Jin-Cherng Chen is with the Vice-Superintendent of Dalin Tzu-Chi General Hospital (e-mail: allen@tzuchi.org.tw). common [3]-[5]. However, without proper treatment and management, the incineration process easily results in the emission of toxic air pollutants; this is because most medical supplies contain plastic materials. [3] argued that because MW generated by medical institutions cannot be effectively controlled and related information is limited, the optimal method for management is to effectively supervise and manage MW from its creation to its final treatment. However, MW pollution does not solely come from medical supplies that are used during the medical treatment process; some are created during the manufacturing process. [4] asserted that the product life cycle can be used to perform a holistic assessment of the influence that various medical supplies have on the environment, and argued that all products impose a certain level of pollution on the environment during each product stage from product manufacture (from raw materials) to transport, use, and final treatment.

In this study, the pollution source was defined as waste created by medical manufacturers during various product stages: raw materials acquisition/manufacturing/product completion, product sales to medical institutions, and product usage by these institutions. The waste created during each of these stages is recognized as industrial waste and companies involved in this process, like companies responsible for creating industrial waste, should be subjected to taxes for the pollution they create, as they bear the responsibility for cleanup. Therefore, the imposition of a pollution tax should be based on the principles of production, consumption, and treatment. However, this complex tax system will inevitably encounter issues that arise because the three product stages differ. Therefore, a simple and basic model was developed, gradually increasing its complexity to meet near real-world application requirements. The theoretical model developed was thus based on simple assumptions.

This study is structured as follows: the first section is an introduction; the second describes the assumptions and notations; the third presents the formulation of the theoretical model; and the fourth presents the conclusion.

\section{Assumptions AND NOTATIONS}

All patients directly or indirectly come into contact with medical supplies during the process of diagnosis, testing, and treatment and these medical supplies ultimately become MW that must be specially treated. Although MW represents a small portion of the total solid waste produced from medical sources, it may retain viruses and other potentially hazardous materials. Failure to effectively control MW may result in human exposure to hazardous environments as well as the polluting of the environment by MW [3]-[6].

Previous studies have primarily focused on MW management, final-stage MW treatment [7], [8], and the risk 
of infection due to MW [9]. In this study, mathematical models were used to test the basic model; the model concepts are shown in Fig. 1. The pollution source was defined as MW created during the various product stages from manufacturing to product sale and usage. The goal was to reduce the effects of pollution by implementing pollution control. This study applied the dynamic optimization method used by [10]; the theory of the model was focused on the factors of sustainable operation and environmental protection. Before formulating the study, several assumptions were made, as described below:

1) In 1987, the Brundlandt Report defined sustainable development as development that meets the needs of the present generation without compromising the ability of future generation to meet their own needs. Therefore, a mathematical model was developed for the medical industry premised on sustainable operation and environmental protection and further discussions were conducted.

2) Unidirectional arrows were drawn in the flow chart to indicate the relationship between variables.

3) For study purposes, certain fixed constants and parameters were given by the Department of Design.

4) No scrap parts were considered during the production process (indicating normal operation and supply).

5) Confounding factors that may have influenced the results such as policy changes were not considered.

The following are the notations used in this study:

$P_{t}$ : Quantity of produced medical supplies

$C_{t}$ : Consumption of medical supplies in hospitals

$T_{X}$ : Pollution tax collected by the government

$G_{1 t}$ : Government expenditure on pollution control

$G_{2 t}$ : Public expenditure by the government to incentivize pollution reduction

$E_{t}$ : Total amount of environmental pollution

$r_{1}$ : Pollution ratio during the manufacturing process

$r_{2}$ : Pollution ratio during the consumption process

$\lambda_{1}$ : Pollution tax imposed on consumers $\left(0<\lambda_{1}<1\right)$

$\lambda_{2}$ : Pollution tax imposed on manufacturers $\left(0<\lambda_{2}<1\right)$

$\lambda_{3}$ : Pollution tax imposed on MW treatment personnel $\left(0<\lambda_{3}<1\right)$

$\theta$ : Ratio between the pollution tax collected by the government and expenditures $(0<\theta<1)$

\section{FORMULATION OF THE THEORETICAL MODEL}

In this section, the notations are defined and the interactions between various variables are depicted. The constant rates and parameters are shown in Fig. 1. Starting from the assumptions, the theoretical mode was formulated.

\section{A. Hospital Consumer $\left(C_{t}\right)$}

Most medical supplies produced by manufacturers are sold through sales channels to hospitals. Therefore, the relationship between the vendors and the hospital were defined as follows:

$$
C_{t}=\left(1-\gamma_{1}\right) P_{t}
$$

where

$r_{1}$ is the waste produced as a ratio to the medical supplies $1>r_{1}>0$, is a constant.

\section{B. MS Treatment Businesses $\left(W_{t}\right)$}

Environmental regulations enacted by the Taiwanese government have instituted strict rules concerning MW treatment. Therefore, it was assumed that hospitals and manufacturers handed over MW and defective products to qualified waste treatment businesses. The relationship between vendors, hospitals, and waste treatment businesses was defined as:

$$
W_{t}=P_{t} \gamma_{1}+C_{t} \gamma_{2}
$$

where

$r_{2}$ is the waste produced as a ratio to the medical supplies $1>r_{2}>0$, is a constant.

\section{Total Pollution Tax Collected by the Government $\left(T_{x}\right)$}

Because the government sets the pollution tax and that pollution sources were assumed created by vendors, hospitals, and MW treatment businesses, the three units were taxed based on the pollution quantity generated. The relationship equation between them is shown as follows:

$$
T_{X}=P_{t} \lambda_{2}+W_{t} \lambda_{3}+\sigma C_{t} \lambda_{1}
$$

where

$\sigma$ is the pollution ratio during the hospital's consumption period.

By substituting (1) and (2) into (3) and reorganizing the equations, (4a) was obtained:

$$
T_{X}=P_{t} \lambda_{2}+\lambda_{3}\left(P_{t} \gamma_{1}+C_{t} \gamma_{2}\right)+\sigma P_{t} \lambda_{1}\left(1-\gamma_{1}\right)
$$

By substituting (1) into (4a), (4b) was obtained:

$$
T_{X}=P_{t}\left(\lambda_{2}+\gamma_{1} \lambda_{3}\right)+\left[P_{t}\left(1-\gamma_{1}\right)\left(\gamma_{2} \lambda_{3}+\sigma \lambda_{1}\right)\right]
$$

The above equations serve as the principles governing the polluters' tax payments. When governments impose a pollution tax, they should contemplate what they are trying to achieve and devise strategies to reduce the amount of pollution. Therefore, it was hypothesized that the pollution tax imposed by the government was used on pollution control (5a) and pollution reduction (5b). Their relationships were defined as:

$$
\begin{gathered}
G_{1 t}=\theta \times T_{x} \\
G_{2 t}=(1-\theta) \times T_{x}
\end{gathered}
$$

where,

$\theta$ is the pollution tax expenditure rate and $1>\theta>0$ is a constant. 
By substituting (4a) into (5a), (6a) was obtained:

$$
G_{1 t}=\theta\left\{P_{t}\left(\lambda_{2}+\gamma_{1} \lambda_{3}\right)+\left[P_{t}\left(1-\gamma_{1}\right)\left(\lambda_{2} \lambda_{3}+\sigma \lambda_{1}\right)\right]\right\}
$$

By substituting (4b) into (5b), (6b) was obtained

$$
G_{2 t}=(1-\theta)\left\{P_{t}\left(\lambda_{2}+\gamma_{1} \lambda_{3}\right)+\left[P_{t}\left(1-\gamma_{1}\right)\left(\lambda_{2} \lambda_{3}+\sigma \lambda_{1}\right)\right]\right\}
$$

\section{Pollution Accumulation $\left(E_{t}\right)$}

Pollution is created when manufacturers manufacture products, when hospitals consume products, and when waste treatment businesses treat waste. The pollution accumulated at time $t$ was assumed to be $E_{t}$, and the relationship equation is shown below:

In this equation, $e_{0}$ is a given constant and is regarded as the initial amount of pollution.

$$
e_{1}=\int_{0}^{t} P_{t} \gamma_{1} d t
$$

$e_{1}>0$ is the pollution accumulated by the manufacturer during manufacturing.

$$
e_{2}=\int_{0}^{t} P_{t} \gamma_{2}\left(1-\gamma_{1}\right) d t
$$

$e_{2}>0$ is the pollution accumulated by the hospitals during consumption.

$$
e_{3}=\int_{0}^{t} P_{t} \gamma_{1}+C_{t} \gamma_{2} d t
$$

$e_{3}>0$ is the pollution accumulated by the waste treatment business during treatment.

By adding $e_{0}$ to $(7 \mathrm{a}),(7 \mathrm{~b})$, and $(7 \mathrm{c})$, the total amount of pollution was obtained (8a)

$$
E_{t}=e_{0}+\left[\int_{0}^{t} P_{t} \gamma_{1}+\int_{0}^{t} P_{t} \gamma_{2}\left(1-\gamma_{1}\right)+\int_{0}^{t} P_{t} \gamma_{1}+C_{t} \gamma_{2}\right] d t \text { (8a) }
$$

When the government invests the pollution tax collected into pollution control, pollution is reduced. The pollution reduction results were hypothesized as follows:

$$
e_{4}=\beta_{1} \int_{0}^{t} G_{1 t} d t
$$

where

$e_{4}>0$ is the accumulation of the government's pollution control results.

$\beta_{1}$ is the pollution control performance ratio, and $\beta_{1}>0$.

Therefore, the government's pollution control efforts produce:

$$
E_{t}=e_{0}+\left[\int_{0}^{t} P_{t} \gamma_{1}+\int_{0}^{t} P_{t} \gamma_{2}\left(1-\gamma_{1}\right)+\int_{0}^{t}\left(P_{t} \gamma_{1}+C_{t} \gamma_{2}\right)-\beta_{1} \int_{0}^{t} G_{1 t}\right] d t
$$

By substituting (6a) into (10a), (10b) was obtained:

$$
\begin{aligned}
E_{t}= & e_{0}+\left[\int_{0}^{t} P_{t} \gamma_{1}+\int_{0}^{t} P_{t} \gamma_{2}\left(1-\gamma_{1}\right)+\int_{0}^{t} P_{t}\left(\gamma_{1}+\gamma_{2}-\gamma_{1} \gamma_{2}\right)\right] \\
& -\beta_{1} \int_{0}^{t} \theta\left[P_{t}\left(\lambda_{2}+\gamma_{1} \lambda_{3}\right)+P_{t}\left(1-\gamma_{1}\right)\left(\lambda_{2} \lambda_{3}+\sigma \lambda_{1}\right)\right] d t
\end{aligned}
$$

The government could also use the pollution tax collected to incentivize pollution reduction, which could lower the amount of pollution created during the manufacturing and consumption stages. The pollution reduction results were hypothesized as follows:

$$
e_{5}=\beta_{2} \int_{0}^{t} G_{2 t} d t
$$

where

$e_{5}>0$ is the pollution reduction accumulated by investing in research and development,

$\beta_{2}$ is the pollution reduction performance ratio, and $\beta_{2}$ $>0$.

$$
E_{t}=e_{0}+\left[\int_{0}^{t} P_{t} \gamma_{1}+\int_{0}^{t} P_{t} \gamma_{2}\left(1-\gamma_{1}\right)+\int_{0}^{t}\left(P_{t} \gamma_{1}+C_{t} \gamma_{2}\right)-\beta_{2} \int_{0}^{t} G_{2 t}\right] d t
$$

By substituting (6b) into (10b), (12b) was obtained

$$
\begin{aligned}
E_{t}= & e_{0}+\left[\int_{0}^{t} P_{t} \gamma_{1}+\int_{0}^{t} P_{t} \gamma_{2}\left(1-\gamma_{1}\right)+\int_{0}^{t} P_{t}\left(\gamma_{1}+\gamma_{2}-\gamma_{1} \gamma_{2}\right)\right] \\
& -\beta_{2} \int_{0}^{t}(1-\theta)\left[P_{t}\left(\lambda_{2}+\gamma_{1} \lambda_{3}\right)+P_{t}\left(1-\gamma_{1}\right)\left(\lambda_{2} \lambda_{3}+\sigma \lambda_{1}\right)\right] d t
\end{aligned}
$$

\section{Decision Model Used to InVEstigate the POLLUTION CONTROL RESULTS}

The study period was a fixed period that began from 0 to $t$. The initial value $e_{0}$ was a given constant, and $P_{t}, C_{t}$, and $E_{t}$ were variables. By rearranging the above equations, the effectiveness that $\mathrm{A}$ and $\mathrm{B}$ had on reducing the total amount of pollution was calculated, where A was the investment in pollution control using the pollution tax collected by the government $\left(G_{1 t}\right)$ and B was public expenditure spent by the government to incentivize pollution reduction $\left(G_{2 t}\right)$. The following three hypotheses were proposed.

Model 1: Assuming that $\beta_{1}>\beta_{2}$, and all of the pollution tax collected was exclusively invested into pollution control, then

\section{1) Objective function}

$$
\begin{aligned}
\max & V_{1}=e_{0}+\left[\int_{0}^{t} P_{t} \gamma_{1}+\int_{0}^{t} P_{t} \gamma_{2}\left(1-\gamma_{1}\right)+\int_{0}^{t} P_{t}\left(\gamma_{1}+\gamma_{2}-\gamma_{1} \gamma_{2}\right)\right] \\
& -\beta_{1} \int_{0}^{t} \theta\left[P_{t}\left(\lambda_{2}+\gamma_{1} \lambda_{3}\right)+P_{t}\left(1-\gamma_{1}\right)\left(\lambda_{2} \lambda_{3}+\sigma \lambda_{1}\right)\right] d t
\end{aligned}
$$

Model 2: Assuming that $\beta_{2}>\beta_{1}$, and all of the pollution tax collected was exclusively invested into pollution reduction incentives, then 


\section{2) Objective function}

$$
\begin{aligned}
\max & V_{2}=e_{0}+\left[\int_{0}^{t} P_{t} \gamma_{1}+\int_{0}^{t} P_{t} \gamma_{2}\left(1-\gamma_{1}\right)+\int_{0}^{t} P_{t}\left(\gamma_{1}+\gamma_{2}-\gamma_{1} \gamma_{2}\right)\right] \\
& -\beta_{2} \int_{0}^{t}(1-\theta)\left[P_{t}\left(\lambda_{2}+\gamma_{1} \lambda_{3}\right)+P_{t}\left(1-\gamma_{1}\right)\left(\lambda_{2} \lambda_{3}+\sigma \lambda_{1}\right)\right] d t
\end{aligned}
$$

Model 3: Assuming that both $\beta_{1}$ and $\beta_{2}$ could effectively reduce pollution and that the government invested some of the pollution tax collected into pollution control and the rest into pollution reduction incentives, then

\section{3) Objective function}

$$
\begin{gathered}
\max V_{1}=e_{0}+\left[\int_{0}^{t} P_{t} \gamma_{1}+\int_{0}^{t} P_{t} \gamma_{2}\left(1-\gamma_{1}\right)+\int_{0}^{t} P_{t}\left(\gamma_{1}+\gamma_{2}-\gamma_{1} \gamma_{2}\right)\right] \\
-\beta_{1} \int_{0}^{t} \theta\left[P_{t}\left(\lambda_{2}+\gamma_{1} \lambda_{3}\right)+P_{t}\left(1-\gamma_{1}\right)\left(\lambda_{2} \lambda_{3}+\sigma \lambda_{1}\right)\right]- \\
\beta_{2} \int_{0}^{t}(1-\theta)\left[P_{t}\left(\lambda_{2}+\gamma_{1} \lambda_{3}\right)+P_{t}\left(1-\gamma_{1}\right)\left(\lambda_{2} \lambda_{3}+\sigma \lambda_{1}\right)\right] d t
\end{gathered}
$$

The amount of pollution accumulated was considered (8a), the government's pollution control results (9a), and the results of its pollution reduction incentives (11a) to derive the objective function of this study. Three possible models were introduced to satisfy the demands of vendors, hospitals, and the government.

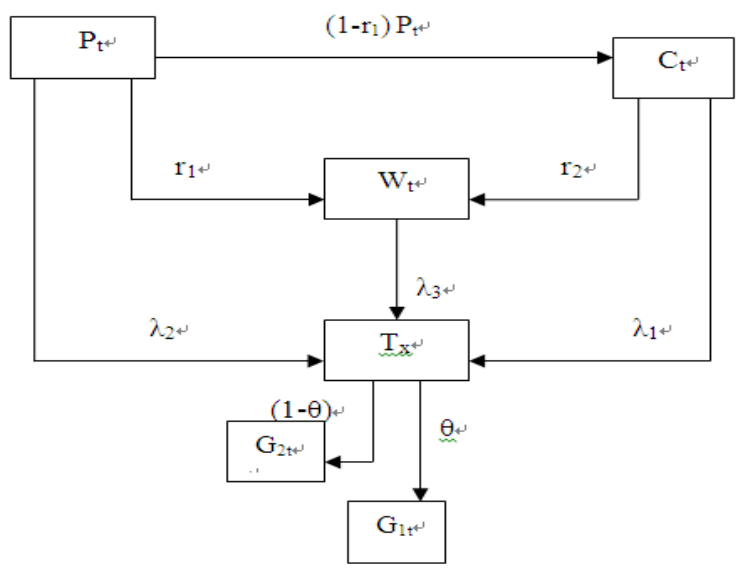

Fig. 1. System flow chart.

\section{CONCLUSION}

In this study, the optimal dynamic theory was used to develop theoretical models premised on sustainable operation and environmental protection. The relationship between the variables was displayed in the flow chart using equations and the fixed parameters and transfer rate conditions were defined. Although the theoretical model differed significantly from those demanded in practical applications, it could nonetheless be used to integrate, analyze, and demonstrate the relationship between the variables, showing the potential for optimized coexistence. A few research contributions can be summarized based on the results of this study. First, concrete data were provided that could be used to discuss the environmental pollution generated by MW. The amount of pollution created by vendors, hospitals, and waste treatment businesses was calculated, and a pollution tax was suggested to be imposed on the three parties by the government to achieve justice. Second, an encouraging approach rather than a punishing one should be used when implementing pollution tax policies, and the pollution tax collected should be used in pollution control to achieve pollution reduction results and protect the environment. The proposed model offers various methods that can be employed in formulating pollution-reduction decisions. Finally, because the model was based solely on mathematical derivation, additional samples should be taken to perform sensitivity analyses to achieve optimized values. These deficiencies should be covered in prospective studies to verify the model's feasibility in practical applications.

\section{REFERENCES}

[1] WHO. Some Basic Information on Healthcare Waste. [Online]. Available: http://www.healthcarewaste.org/en123_hcw_general.html.

[2] M. A. Habash and A. A. Zu'bi, "Efficiency and effecteviness of medical waste management performance, health sector and its impact on environment in Jordon applied study," World Applied Sciences Journal, vol. 19, no. 6, pp. 880-893, 2012.

[3] Y. C. Jang, C. Lee, O. S. Yoon, and H. Kim, "Medical waste management in Korea," Journal of Environmental Management, vol. 80 , no. 2, pp. $1-9,2005$

[4] B. Kaiser, P. D. Eagan, and H. Shaner, "Solutions to health care waste: life-cycle thinking and green purchasing," Environmental Health Perspectives, vol. 109, no. 3, pp. 205-207, 2001

[5] S. Mohankumar and K. Kottaiveeran, "Hospital Waste Management and Environmental Problems in India," International Journal of Pharmaceutical and Biological Archives, vol. 2, no. 6, pp.1621-1626, 2011.

[6] D. S. CE, H. AE, R. MM, and N. Mello, "Medical wastes management in the south of Brazil," Waste Management, vol. 25, pp. 600-605, 2005.

[7] C. C. Lee and G. L. Huffman, "Medical waste management/incineration," Journal of Hazardous Materials, vol. 48, pp. 1-30, 1996.

[8] L. B. Sztanyik, "A review of the management of radioactive wastes in medical institutes," Waste Management and Research, vol. 11, no. 5, pp. 429-439, 1993.

[9] R. R. M. Gershon, C. Karkashian, D. Vlahov, M. Grimes, and E. Spannhake, "Correlates of infection control practices in dentistry," American Journal of Infection Control, vol. 26, no. 1, pp. 29-34, 1998.

[10] M. I. Kamien and N. L. Schwartz, Dynamic Optimization-The Calculus of Variations and Optimal Control in Economics and Management $4^{\text {th }}$ edition, North Holland Inc. N.Y.1981.

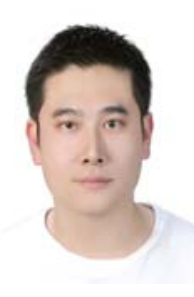

Chen-Lin Lee is a PhD candidate in Department of Business Administration in Nanhua University in Taiwan. He also is the chief of Inventory Department of Dalin Tzu-Chi Hospital. His research interests lie in the fields of human resource management, medical material management and information management.

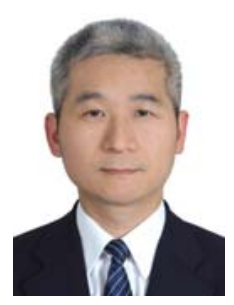

Jin-Cherng Chen is the vice-superintendent of Buddhist Dalin Tzu-Chi Hospital. His research interests are focused on the fields of brain tumor, neuroepithelioma. He has published many international journal papers regarding the above-mentioned fields. 